\title{
Enhancing technology of producing secure IOT devices on the base of remote attestation
}

\author{
Vasily Desnitsky * and Igor Kotenko \\ St. Petersburg Federal Research Center of the Russian Academy of Sciences (SPC RAS), \\ St. Petersburg Institute for Informatics and Automation of the Russian Academy of Sciences, 39, \\ 14th Liniya, 199178, St. Petersburg, Russia
}

\begin{abstract}
The goal of the work is to enhance the technological process for the production of components of integrated secure systems of the Internet of Things for solving problems of operational control and reaction in emergency situations. The most important requirement for such systems is the need to ensure the properties of reliability and security of software and hardware elements of the end devices, taking into account the specificity of such systems. To achieve the goal in the paper the mechanisms for protection of Android applications from the threats of integrity violation of the software and of critical data on the base of remote attestation principles are modeled. Analytical and experimental evaluations of the implemented protection components and the protocol of their interaction taking into account limitations on the computing and communication resources of the target device are performed.
\end{abstract}

\section{Introduction}

A problem of protecting of systems for operational control and reaction in emergency situations from unauthorized modification threats is becoming increasingly important and is caused by the susceptibility of software platforms of mobile and embedded devices such as Android, Raspberry Pi and others to threats of integrity and authenticity violation of the code and data used. To solve this problem it is necessary to develop mechanisms for embedding protection means within the technological process of producing such systems.

In general application of algorithms controlling immutability of the software, which are built directly into the program they protect, can increase the protection level. However the local nature of the protection and limiting its persistence as well as situating the program in an environment being non-trusted and uncontrolled by the software developer or the owner of the digital rights leads to the fact that such protection mechanisms can be neutralized by an intruder if there are sufficient tools and resources.

The remote validation mechanism, investigated in the paper, is based on the use of a client-server approach to protection and allows increasing security of the software under resource limitations of the mobile platform as well as limitations of the communication channel bandwidth.

*Corresponding author: desnitsky@comsec.spb.ru 
Fig. 1 shows the main stages of the technological process of producing the components of an integrated protected system of operational control and reaction in emergency situations.

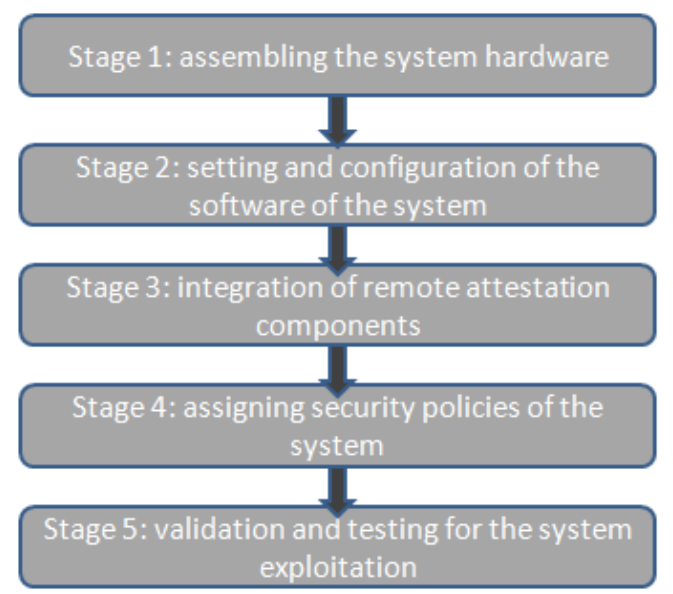

Fig. 1. The main stages of the technological process of producing the target system.

The expansion of the technological process for producing secure systems for operational control and reaction in emergency situations includes the stage of forming a cloud based computing structure responsible for verifying remote devices by using a client-server approach and building a secure communication protocol.

In the paper modeling and analysis of particular protection algorithms are performed within the framework of an integrated approach to the implementation of software protection components, implementing remote attestation with the use of Android platform.

The distinguishing features of the results achieved in the paper include, in particular, experimental data obtained during the modeling of protection components under mobile operating system limitations.

The paper is organized as follows. Section 2 provides an overview of existing works in the subject field. Section 3 reveals features of the approach to remote attestation of mobile applications. Section 4 describes results of modeling of specific remote attestation based algorithms that implement. Section 5 presents results of the experimental studies, whereas Section 6 concludes the paper.

\section{Related work}

Brasser et al. [1] and C.Preschern et al. [2] consider remote attestation as means of protection against malicious software intrusion attacks on embedded devices [3]. The features and methods that allow implementation of the attestation with minimal additional costs are demonstrated. At that C.Preschern et al. [2] propose adaptation of software methods for remote attestation to solve tasks of protecting critical systems with minimal forced revision of established procedures for safety properties certification.

J.Ho et al. [4] show that in sensor networks the remote certification is used for detection of self-propagating network worms by sequentially infecting nodes using traffic detection methods.

Srinivasan et al. [5] investigate remote software-based attestation to ensure the integrity of the operating system kernel and user applications. In particular the authors propose a technique that allows determining whether the already certified application was substituted by an intruder or not. 
M. Santra, et al. [6] propose the use of remote attestation and the three-phase protocol constructed on it, using a SELinux module for providing secure interaction in distributed information systems. The paper also substantiates effectiveness of the proposed approach, using methods of formal analysis and ProVerif verifier.

T. Abu Hmed, et al. [7] propose software techniques for remote attestation of wireless sensor networks against tampering attacks into their work. These techniques are not based on the use of the accuracy factor of the measured runtime execution, thereby improving previously proposed integrity monitoring methods in wireless sensor networks [8].

D. Fu and X. Peng [9] analyze security of the mechanisms of one- and multi-hop attestation in wireless sensor networks.

Tan et al. [10] propose a multi-level remote attestation protocol to monitor integrity of IoT-systems, taking into account their inherent computational limitations and device's power limitations.

In [11], [12] the authors propose a reference architecture and partial models for mechanisms of IoT remote attestation, using cloud solutions to improve the targets of the remote attestation process.

K. Ramachandran and H. Lutfiyya [13] prove the importance of remote attestation of software updates, using cloud computing and procedures for verifying its correctness [14].

Y.Zhang et al. [15] extend remote attestation application to ensuring the confidentiality by a modified Extended Hash Algorithm [16]. It allows increasing level of the confidentiality with comparable performance characteristics during the execution.

T.Syed et al. [17] propose effective solutions for increasing scalability of mechanisms for remote attestation of device sets by using Big Data technology, including using multiprocessor systems [18], property based authentication mechanisms [19] and characteristics of these properties [20].

Increasing the efficiency of the server part of the authentication mechanism with a large number of instances of attested programs is also achieved by reorganizing and reducing the chain of trust used in the attestation process [21].

$\mathrm{H}$. Li et al. [22] propose models of remote attestation based on the paradigm of attack graphs to tackle tasks of monitoring and attestation of software components [23].

\section{Approach to remote attestation of mobile applications}

Remote attestation of a mobile application includes software local and remote components that are located within a non-trusted and trusted environment, respectively, as well as a secure protocol for their network interaction.

The interaction between the components is based on roles of a client the attested entity, and the server the attesting one. The protocol assumes implementation of the protection functions of the protocol itself from possible interception and modification of packets at the transport level. The payload of the protocol includes program identifiers and numeric values that characterize the current state of elements of the program code and critical data of the application.

Specific algorithms used within the framework of the protection mechanism on the base of remote attestation principles assume, first, introduction of specific constructions emplaced into the objective code at the stage of forming the syntactic tree of the application and, second, isolation of basic blocks and particular instructions in the code.

Security constructions do not directly perform any code integrity checks and data locally, but send their snapshots to the side of the trusted server. This fact greatly complicates successful intruder's modification of the application, being not subsequently detected on the server side. 
A typical scenario for applying remote attestation to the protection of mobile devices involves remote control by a mobile application store or content provider over multiple instances of client applications. In case of a violation it warns on the violation on a specific device and stops its further maintenance until the detected violation is rectified.

\section{Protection algorithms}

A control flow checking algorithm is based on a control flow graph of the protected program, built statically. The graph is used in dynamics for remote control of the correctness of the process of its execution. This algorithm allows ensuring the correctness of the execution of a sequence of commands, including branching structures, loops, handling of exceptional situations, etc.

The control flow checking algorithm includes two stages, namely static and dynamic. Statically one prepares and embeds the attesting module constructions in the program code. The initiating construction establishes a connection to the remote attesting module via HTTP sockets.

Program markers, which are operations send (A) of sending a specific identifier A to the server side, are situated in the program code on the boundaries of the base blocks. The attesting module function on the client side contains sending a sequence of identifiers of program markers during their passage in the execution process (dynamically).

On the server side of the connection one constructs a regular expression, which determines the correct chains of operation of program markers within the static stage. The regular expression is used to build a transition graph, which nodes determine program markers and arcs denote permissible transitions between them.

At the dynamic stage when receiving the identifiers of program markers from the client side the process of traversing the graph is performed and its correctness is checked in accordance with the structure of the graph.

As an example Fig. 2 schematically shows a fragment of the code of the protected program with built-in functions for sending program markers.

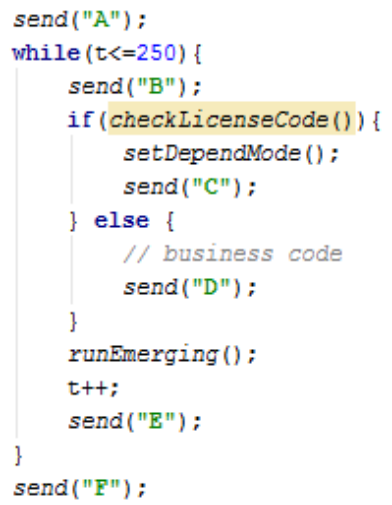

Fig. 2. Code fragment of the protected program.

The regular expression in the infix form constructed for the given fragment on the server side is $A(B(C / D) E)^{*} F$. The graph of transitions with the final vertex $F$ corresponding to this regular expression is shown in Fig. 3. 


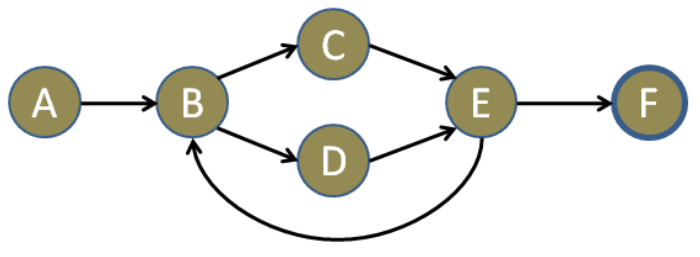

Fig. 3. Transition graph for the remote attestation algorithm.

The checksum algorithm requires the existence of invariant data structures that are critically important in relation to the task of ensuring the integrity of the protected code and data. Cryptographic algorithm MD5 is used as the basis.

The sending to the server side of the connection and checking the received token is done by using the send md5(criticulStructure) and verify(getNextToken()) functions, respectively.

\section{Experiments and discussion}

The evaluation of the solutions constructed in the work is done by defining and analyzing values of a number of indicators, namely, the indicators of efficiency, reliability and resource consumption [24].

The efficiency indicator is due to the hardware limitations of the Android platform and the limited bandwidth of the communication channel, which affect the stability and continuity of the application, as well as the usability of the end user.

The efficiency is calculated on a test scenario by using a system function System.currentTimeMillis() as an average value of the time delays that occur as a result of executing instructions for sending program markers and checksums.

The results of the conducted experiments showed that when the ratio of the number of built-in instructions of the evaluating module to the number of instructions of the target program not exceeding $20 \%$, the average delay value did not exceed the established allowable limit of $200 \mathrm{~ms}$.

Calculation of the resource consumption indicators is performed by using the jmap and jstat utilities. These ones allow estimating the increase in the consumption of the consumed RAM on the client side after adding the attestation functions to the code. Based on a series of measurements made on the test application, it was determined that the increase in the memory consumption did not exceed $21 \%$ in comparison with the unprotected version of the software application.

To evaluate the reliability indicator of the proposed security solution, fuzzy testing of the protected application was performed on pre-generated tests, including random and boundary values of the input data. Testing a series of 250 samples of input data revealed no false positive and false negative errors. Therefore it confirms the correctness of the proposed approach to the remote attestation and the operational capability of its software implementation.

Applicability of the proposed approach to protection of the integrity of Android applications is due to the achievable level of deployment automation of the proposed security solutions as well, including the choice of location and placement of attesting instructions in the code. This makes it possible to solve the problems of efficient selection and adaptation of existing software tools for processing Java code, both at the source code level and directly by using bytecode analysis tools.

The experiments on a test bench using ZigBee Series 2 microcontrollers have confirmed the applicability of the used extended technological process for the production of systems for operational control and reaction in emergency situation in practice. 
The experiments performed also confirmed that the expected effect of the implementation of the results obtained is, first, in improving the security indicators of the final operational control and reaction system in emergency situations, and, second, in expanding the providing functionality of the system by introducing the stage of integrating remote certification in the used technological process.

\section{Conclusion}

An approach to remote attestation of mobile applications, using control flow checking and checksum checking algorithms has been investigated.

Within the proposed and tested technological process a hardware/software implementation of the algorithms was performed by using Android platform as an example to serve as a basis for obtaining experimental characteristics of these algorithms.

As a direction for future research it is planned, first, to develop techniques to analyze security of mobile applications and, second, to increase their security, including at the source code level and object code one.

The work is supported by RFBR (project No. 19-07-00953).

\section{References}

1. F. Brasser, K. B. Rasmussen, A. R. Sadeghi, G. Tsudik, Remote attestation for low-end embedded devices: The prover's perspective, in Proceedings of 53nd

ACM/EDAC/IEEE Design Automation Conference (DAC), Austin, TX, 1-6 (2016)

2. C. Preschern, A. J. Hörmer, N. Kajtazovic, C. Kreiner, Software-Based Remote Attestation for Safety-Critical Systems, in Proceedings of 2013 IEEE Sixth

International Conference on Software Testing, Verification and Validation Workshops, Luxembourg, 8-12 (2013)

3. V. Desnitsky, A. Chechulin, I. Kotenko, Levshun D., M. Kolomeec, Combined Design Technique for Secure Embedded Devices Exemplified by a Perimeter Protection System, SPIIRAS Proceedings, 48, 5-31 (2016)

4. W. Ho, M. Wright, Distributed Detection of Sensor Worms Using Sequential Analysis and Remote Software Attestations, IEEE Access, 5, 680-695 (2017)

5. R. Srinivasan, P. Dasgupta, T. Gohad, Software Based Remote Attestation for OS Kernel and User Applications, in Proceedings of IEEE Third International Conference on Privacy, Security, Risk and Trust and IEEE Third International Conference on Social Computing, Boston, MA, 1048-1055 (2011)

6. M. Santra, S. K. Peddoju, A. K. Bhattacharjee, A. Khan, Design and Analysis of a Modified Remote Attestation Protocol, in Proceedings of IEEE Trustcom/BigDataSE/ICESS, Sydney, NSW, 578-585 (2017)

7. T. AbuHmed, N. Nyamaa, D. Nyang, Software-Based Remote Code Attestation in Wireless Sensor Network, in Proceedings of IEEE Global Telecommunications Conference (GLOBECOM 2009), Honolulu, HI, 1-8 (2009)

8. K. Xiangying, C. Yanhui, Dynamic Remote Attestation Based on Concerns, in Proceedings of 8th International Symposium on Computational Intelligence and Design (ISCID), Hangzhou, 76-80 (2015)

9. D. Fu, X. Peng, TPM-based remote attestation for Wireless Sensor Networks, Tsinghua Science and Technology, 21(3), 312-321 (2016) 
10. H. Tan, G. Tsudik, S. Jha, MTRA: Multiple-tier remote attestation in IoT networks, in Proceedings of IEEE Conference on Communications and Network Security (CNS), Las Vegas, NV, 1-9 (2017)

11. H. Song, G. A. Fink, S. Jeschke, Secure Registration and Remote Attestation of IoT Devices Joining the Cloud: The Stack4Things Case of Study, Security and Privacy in Cyber-Physical Systems: Foundations, Principles, and Applications, 1, Wiley-IEEE Press, 137-156 (2017)

12. S. Azadiabad, H. Pedram, M. R. Abbasy, Scalable protocol for remote integrity attestation of cloud based distributed services, in Proceedings of IEEE 8th International Conference on Application of Information and Communication Technologies (AICT), Astana, 1-5 (2014)

13. K. Ramachandran, H. Lutfiyya, A remote attestation infrastructure for verifying the application of software updates, in Proceedings of IFIP/IEEE Symposium on Integrated Network and Service Management (IM), Lisbon, 317-325 (2017)

14. V. Desnitsky, I. Kotenko, Expert Knowledge based Design and Verification of Secure Systems with Embedded Devices, in Proceedings of 4rd IFIP International Workshop on Security and Cognitive Informatics for Homeland Defense (SeCIHD 2014), Fribourg, Switzerland, Lecture Notes in Computer Science (LNCS), vol. 8708, Springer-Verlag, 194-210 (2014)

15. Y. Zhang, L. Wang, Y. You, L. Yi, A Remote-Attestation-Based Extended Hash Algorithm for Privacy Protection, in Proceedings of International Conference on Computer Network, Electronic and Automation (ICCNEA), Xi'an, 254-257 (2017)

16. K. Xiangying, C. Yanhui, Left full binary hash tree for remote attestation, in Proceedings of IEEE 2nd International Conference on Signal and Image Processing (ICSIP), Singapore, 385-390 (2017)

17. T. A. Syed, S. Jan, S. Musa, J. Ali, Providing efficient, scalable and privacy preserved verification mechanism in remote attestation, in Proceedings of International Conference on Information and Communication Technology (ICICTM), Kuala Lumpur, 236-245 (2016)

18. M. Kiperberg, A. Resh, N. J. Zaidenberg, Remote Attestation of Software and Execution-Environment in Modern Machines, in Proceedings of IEEE 2nd International Conference on Cyber Security and Cloud Computing, New York, NY, 335-341 (2015)

19. Y. Liang, K. E. Guo, J. Li, The remote attestation design based on the identity and attribute certificates, in Proceedings of 11 th International Computer Conference on Wavelet Actiev Media Technology and Information Processing (ICCWAMTIP), Chengdu, 325-330 (2014)

20. A. Francillon, Q. Nguyen, K. B. Rasmussen, G. Tsudik, A minimalist approach to Remote Attestation, in Proceedings of Design, Automation \& Test in Europe Conference \& Exhibition (DATE), Dresden, 1-6 (2014)

21. W. Luo, W. Liu, Y. Luo, A. Ruan, Q. Shen, Z. Wu, Partial Attestation: Towards CostEffective and Privacy-Preserving Remote Attestations, in Proceedings of IEEE Trustcom/BigDataSE/ISPA, Tianjin, 152-159 (2016)

22. H. Li, S. Wang, An Efficient and Flexible Dynamic Remote Attestation Method, in Proceedings of Ninth International Conference on Broadband and Wireless Computing, Communication and Applications, Guangdong, 239-246 (2014) 
23. C. Meng, Y. He, Q. Zhang, Remote Attestation for Custom-built Software, in Proceedings of International Conference on Networks Security, Wireless Communications and Trusted Computing, Wuhan, Hubei, 374-377 (2009)

24. V. Desnitsky, I. Kotenko, Modeling and Analysis of IoT Energy Resource Exhaustion Attacks, in Proceedings of 11th International Symposium on Intelligent Distributed Computing (IDC'2017), Intelligent Distributed Computing XI, Studies in

Computational Intelligence, Springer-Verlag, vol.737, Belgrade, Serbia, 263-270 (2017) 